\title{
Effects of End-Terminal Capping on Transthyretin (105-115) Amyloid Protofibrils Using Steered Molecular Dynamics
}

\author{
Myeongsang Lee, Hyunsung Choi, and Sungsoo Na \\ Department of Mechanical Engineering, Korea University, Seoul 136-701, Republic of Korea \\ Correspondence should be addressed to Sungsoo Na; nass@korea.ac.kr
}

Received 30 December 2015; Revised 31 March 2016; Accepted 10 April 2016

Academic Editor: Zhiping Xu

Copyright ( 2016 Myeongsang Lee et al. This is an open access article distributed under the Creative Commons Attribution License, which permits unrestricted use, distribution, and reproduction in any medium, provided the original work is properly cited.

\begin{abstract}
Numerous degenerative diseases are associated with amyloidosis, which can be caused by amyloid proteins. These amyloid proteins are generated from misfolded and denatured amyloid monomers under physiological conditions. Changes in protonation state, $\mathrm{pH}$, ionic strength, and temperature, in addition to mutations, are related to the promotion of amyloidosis. Specifically, an understanding of the mechanical characteristics of amyloid protofibrils is important, since amyloid growth proceeds by a mechanism involving cycles of fragmentation and elongation. However, there remains a lack of knowledge of amyloid structural conformations and their mechanical characteristics, particularly considering end-terminal capping effects. In the present study, we investigated the mechanical characteristics of transthyretin amyloid protein (TTR), which have been implicated in cardiovascular disease, and specifically considered the contribution of end-terminal capping effects. Using steered molecular dynamics (SMD) simulations, we report different structural behaviors between uncapped and capped TTR amyloid protofibrils. We show that end-terminal capping strengthens the structural stability and improves the mechanical properties of amyloid protofibrils. This study provides useful information concerning the structural and mechanical characteristics of TTR amyloid protofibrils, with a particular emphasis on end-terminal capping effects.
\end{abstract}

\section{Introduction}

Many of the major types of degenerative disease are associated with denatured and misfolded amyloid proteins [1-3]. For example, type II diabetes has been associated with human islet amyloid polypeptide (hIAPP), since hIAPP triggers betacell death in pancreas [4]. As for dialysis-related degenerative diseases, beta-2-microglobulin amyloid proteins are found in the joints of patients due to long-term hemodialysis treatments $[5,6]$. Moreover, transthyretin (TTR) amyloid proteins can contribute to cardiovascular disease by narrowing cardiac vasculature $[7,8]$. The phenomenon shared by these degenerative diseases is the deposition of amyloid proteins to normal functional cells, where they are hard to degrade under physiological conditions $[9,10]$.

These amyloid proteins exist in several forms such as monomers, oligomers, fibrils, and plaques. A specific understanding of oligomeric amyloid fibrils is crucial, due to their toxic characteristics [11-14]. Such toxicity was established by several experimental studies, and it the results of amyloid toxicity were the generation of ion channels across membranes, removal of lipid layers from membranes, permeation of oligomers to membranes, and additional growth of amyloid fibrils [11]. Specifically, investigations have highlighted that the size of oligomeric protofibrils is a critical parameter, since the fragmentation and elongation mechanism is related to fibrillar size. According to Collins et al., they experimentally investigated the amyloid growth mechanism using prion protein Sup 35 via three step models (i.e., nucleation, monomer addition, and fiber fragmentation) [15]. Based on this point, fundamental computational and experimental studies on fracture phenomena of amyloid fibrils may provide mechanical insights $[10,16]$.

Detailed computational studies have been conducted on amyloid protofibrils, examining the polymorphic characteristics [17-21], point-mutation effects [22-24], and amyloid fibrils of differing cross-sections under physiological conditions [25-27]. Paparcone et al. have provided mechanical characterization of $\mathrm{A} \beta$ fibrils, which are associated with Alzheimer's disease [23]. They varied the salt bridge region 
and examined effects on structural stability of mutating lysine and aspartate residues for each. In similar manner, Chang et al. found via molecular dynamics (MD) that the effect of mutating residues depends on their being located within hydrophobic core of $\mathrm{A} \beta$ [22]. Ndlovu et al. [28, 29] and Yoon et al. investigated the effects of polymorphic characteristics and point mutations on hIAPP using equilibrated MD and steered molecular dynamics (SMD) $[19,30]$. In their studies, they investigated the effects of altering the orientation of betastrands and the effect of mutating leucine residues to phenylalanine residues on structural conformation and mechanical characteristics. In these computation studies, structural stabilities and mechanical properties of fibrillar amyloid proteins have been reported, in order to better understand the mechanisms of fragmentation under physiological conditions.

Recently, several groups considered whether endterminal capping or altered protonation state have an effect on the structural characteristics of amyloid fibrils associated with degenerative and neurodegenerative diseases [31-34]. For example, the effects of capping hIAPP amyloid fibrils were studied experimentally and computationally. Andreasen et al., demonstrated that different conformations and structural characteristics of hIAPP fibrils are dependent on terminal capping (i.e., N-terminal acetylation and C-terminal amidation) by a combination of transmission electron microscopy (TEM), atomic force microscopy (AFM), and MD methods [32]. Specifically, they investigated the molecular characteristics of hIAPP amyloid proteins arising from terminal capping effects via twisting angle between each beta-strand and the number of hydrogen bonds. Porrini et al. used MD simulations to consider the effect of different protonation states on the oligomeric characteristics of amyloidogenic peptide derived from TTR 105-115 [31]. Different structural characteristics were observed depending on the protonation state, including different beta-strand content and altered twisting angles and the root mean square deviations (RMSDs). In similar manner, Lee and Na recently investigated the structural characteristics of TTR 105-115 amyloid protofibrils by end-terminal capping effects using equilibrated MD simulation methods [35]. However, the effect of capping on mechanical characteristics of TTR amyloid protofibrils remained unstudied using SMD methods.

In this study, we report the mechanical characteristics and material properties of end-terminal capped and uncapped TTR 105-115 amyloid protofibrils, using SMD methods. During the SMD simulation, we noticed a considerably stronger reaction force from the capped fibrils than from uncapped fibrils. We also report Young's modulus of the capped TTR amyloid protofibrils, and we found a larger number of hydrogen bonds in the capped fibrils. Using our SMD simulation, we also calculated the variation in electrostatic force due to end-terminal capping.

\section{Material and Methods}

2.1. Computational Model Construction for the SMD Simulation. In order to investigate the mechanical characteristics of capping effect on TTR amyloid protofibrils, we used the computational results of capped and uncapped TTR amyloid protofibrils from our previous study [35]. Basically, the atomic coordinates of TTR amyloid protofibrils contained in the protein data bank (PDB-ID: $2 \mathrm{M} 5 \mathrm{~N}$ ) were used as a building block for a protofibrils [36]. Geometrical conditions for protofibrils construction were also same as those in the previous experimental studies which were revealed by Dobson's group [36-38].

From our previous study [35], autopsf plugin of VMD 1.9.1 was used to give the capping effect on TTR amyloid protofibrils, by acetylating the $\mathrm{N}$-terminus and amidating the C-terminus of the modeled fibrils [39]. Equilibrium simulation of each capped and uncapped protofibril was computed using NAMD package 2.8 [40] with CHARMM27 force field. Each protofibril model was minimized over 10,000 steps via the conjugate-gradient method. Subsequently, protofibrils were solvated in a TIP3P water box without counter ions because TTR amyloid protofibrils carried no net charges. Additionally, a minimum border-distance of $20 \AA$ was applied from each side of the solvent box.

The MD simulations were performed for $50 \mathrm{~ns}$ after the simulated models to reach local minima on the potential energy surfaces. MD simulations were equilibrated at a constant-temperature $(298 \mathrm{~K})$ and constant-volume condition. Equilibrated results of both uncapped and capped TTR amyloid protofibrils are confirmed through the root mean square deviation and their twist angle between each beta-strands along the fibrils axis from previous study [35]. After that, the final coordinates of capped and uncapped protofibrils were obtained to use in a steered molecular dynamics (SMD) simulation in this study. Obtained capped and uncapped TTR fibrils after equilibrated MD simulations are shown in Figure 1.

\subsection{Steered Molecular Dynamics (SMD) Simulation Proto-} col. To analyze the different mechanical behavior between uncapped and capped TTR amyloid protofibrils, SMD simulations were performed to measure tensile properties. The same NAMD package 2.8 and CHARMM27 force field was used in the SMD simulations [40]. The constant-temperature $(298 \mathrm{~K})$ and constant-volume ensemble was also adopted during the SMD simulations, until fracture of the fibrils occurred. The spring constant was $7.2 \mathrm{kcal} / \mathrm{mol} \cdot \AA^{2}$, where $1 \mathrm{kcal} / \mathrm{mol}$ equates to $69.489 \mathrm{pN} \cdot \AA$, throughout the entirety of the simulations. As shown in Figure 2, the two bottom layers were fixed, and the two top layers were pulled at several constant velocities, such as $0.001 \AA /$ ps and $0.005 \AA /$ ps. Reaction forces were calculated using $F(t)=K_{\text {spring }}(v t-X)$, where $v$ is a pulling speed, $t$ is time, and $X$ is a displacement variable. The resulting relationship between the reaction forces and displacements for pulling rate was obtained after the SMD simulations. Initial fibril lengths and cross-sectional areas, for both capped and uncapped fibrils, were calculated by computing stress-strain relationships.

2.3. Calculating the Mechanical Characteristics and Properties of TTR Protofibrils. For the analysis of the mechanical 


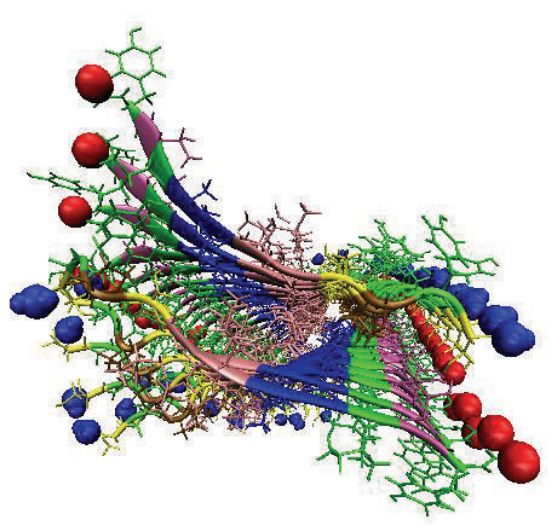

Capped model

(a)

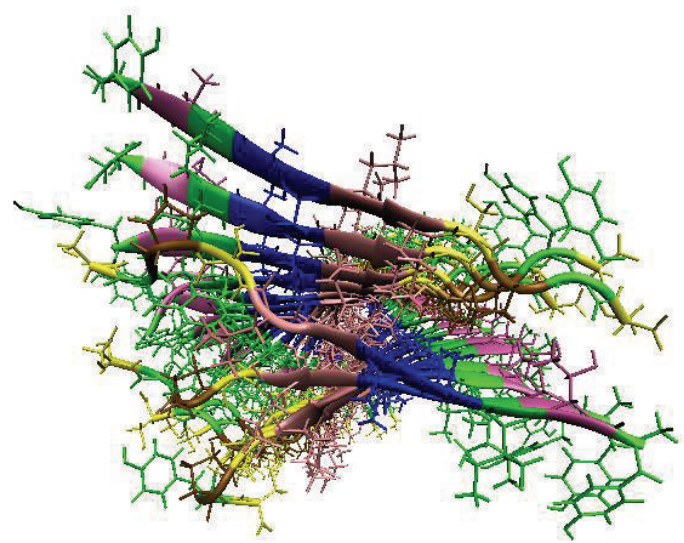

Uncapped model

(b)

FIGURE 1: A schematic image of TTR amyloid protofibrils depends on end-terminal capping. (a) A model of a capped fibril. The capped region is represented with red and blue spheres. (b) A model of an uncapped fibril for the simulation.

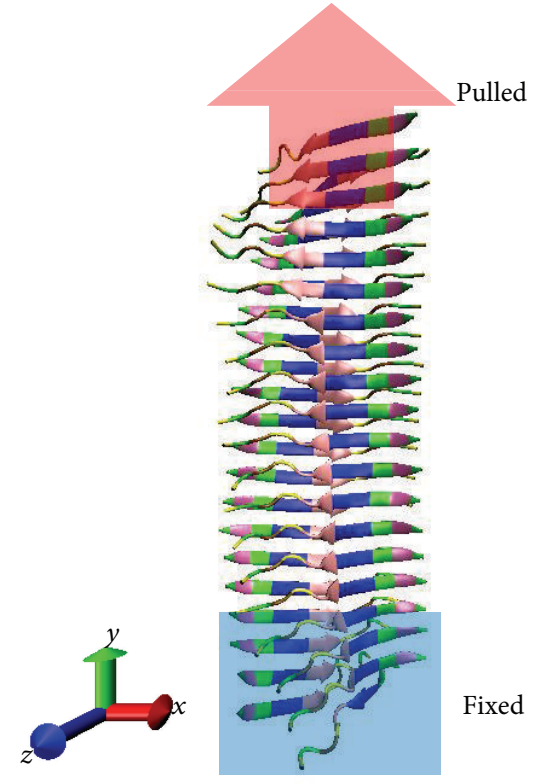

FIGURE 2: A constraint for the tensile simulations, computed by SMD simulation. The blue boxed region of TTR amyloid protofibril was fixed. The red boxed area, corresponding to the top two layers of the protofibril, was pulled along the fibril axis, which is parallel to $y$-axis.

characteristics of TTR amyloid protofibrils, classical beam theory was used to calculate Young's modulus for each case. The TTR protofibrils were regarded as beam models. The constitutive relation is given by Hooke's law, which represented as $\sigma_{y y}=E \cdot \varepsilon_{y y}$. Here, $\sigma_{y y}$ is the stress along the fibril axis which is parallel to $y$-axis (Figure 1), $E$ is Young's modulus, and $\varepsilon_{y y}$ is the strain along to the fibril axis (i.e., $z$-axis). Stress could be expressed as $\sigma_{y y}=F / A$. Here, $F$ is the calculated force during the SMD simulation, and $A$ is the measured cross-sectional areas, where we assumed the cross-section area of TTR amyloid protofibrils as rectangular shapes. Four alpha carbons located on the end-terminus of two beta-strands of bottom layer are regarded as edges of rectangular shape from cross-section of TTR amyloid protofibrils. Therefore, the cross-sectional area was calculated by measuring the width and height from rectangular shape. Stress was computed by calculating the tensile loading on the cross-sectional area of fibrils over time, and strain variation was obtained by dividing the change in length of a fibril during the same time by its initial length $(\sim 10 \mathrm{~nm})$.

\section{Results and Discussion}

3.1. Different Fracture Behavior of TTR Amyloid Protofibrils Caused by End-Terminal Capping. Our previous study, using a 50 ns equilibrated MD simulation and principle component analysis (PCA), examined that $\mathrm{N}$-terminal acetylation and C-terminal amidation of TTR amyloid protofibrils alter their structural conformations and mechanical characteristics [35]. Based on those results, we reported considerably improved structural stabilities and Young's moduli of capped TTR amyloid protofibrils. Differences in Young's modulus values were two times larger than the excepted torsional modulus.

In this study, employing constant-velocity loading simulations, we showed that TTR 105-115 amyloid material strength is different, depending on end-terminal capping status. Further, in order to detect end-terminal capping effects in detail, we applied two different loading conditions $(0.001 \AA / p s$ and $0.005 \AA / p s)$. As shown in Figures 3(a)-3(d), in both $0.001 \AA /$ ps and $0.005 \AA /$ ps constant-velocity loading conditions, we found higher peak forces for capped protofibrils. Under $0.001 \AA /$ ps constant-velocity loading simulations (see Figures 3(a) and 3(b)), the first peak force events for both uncapped and capped TTR were observed around the $1 \mathrm{~ns}$ period. At the peak force value, however, both the rupture 

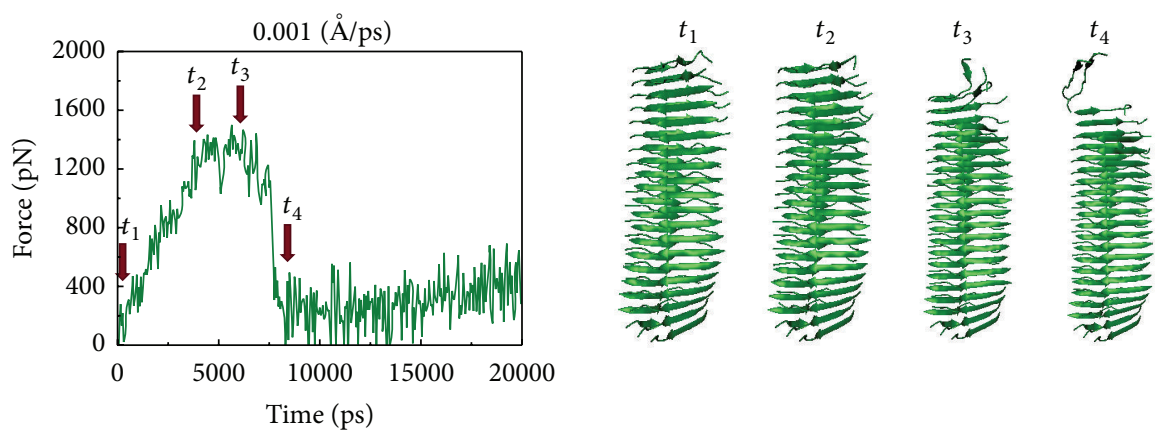

- Capped

(a)
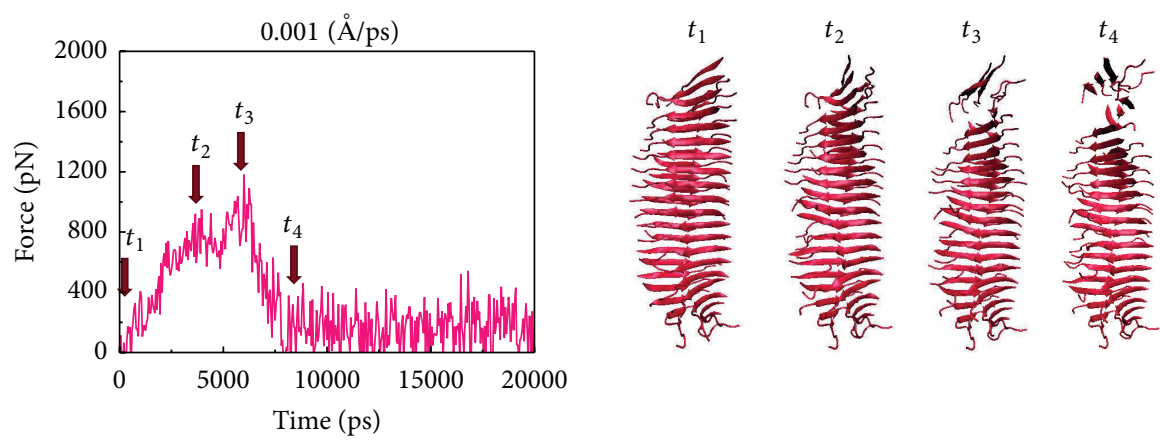

- Uncapped

(b)
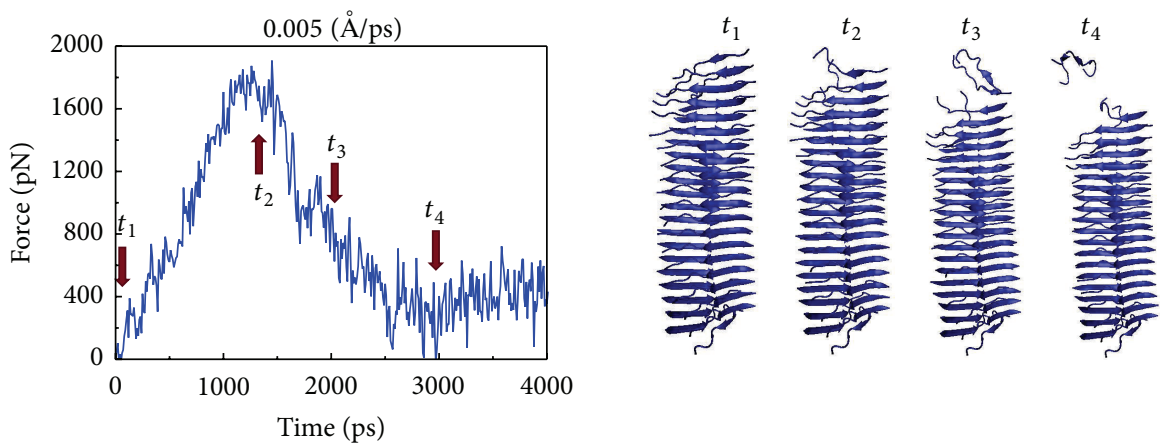

— Capped

(c)
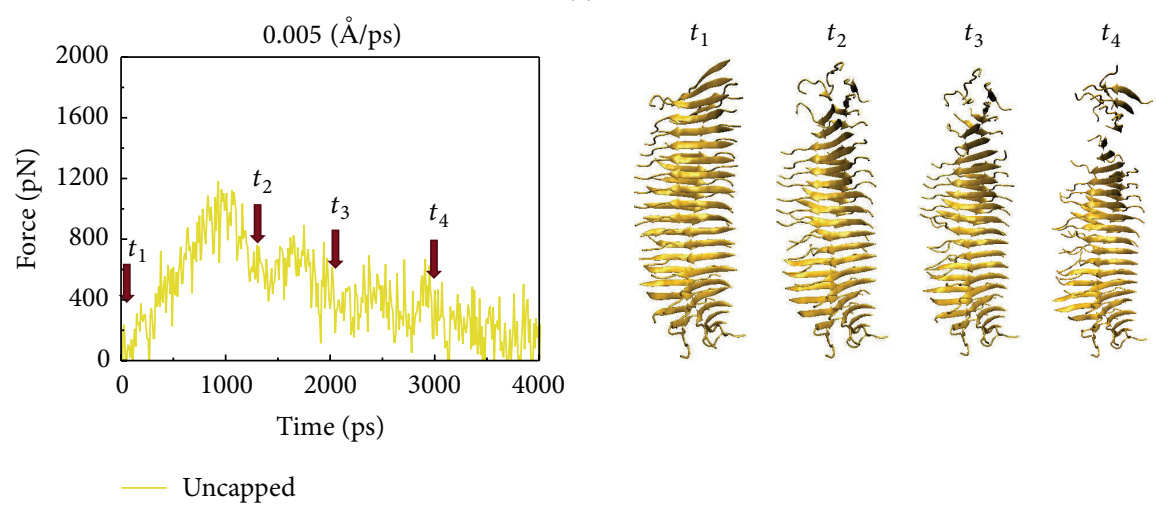

(d)

FIGURE 3: Time versus force graphs comparing different fracture features of capped and uncapped fibrils for time. Both (a) and (b) show the results of SMD simulation with a pulling rate of $0.001 \AA / \mathrm{ps}$ for the capped model and the uncapped model, respectively. Panels (c) and (d) show the results for the capped model and the uncapped model, respectively, at a pulling rate of $0.005 \AA$ ps. Fracture processes over time are indicated by the downward arrows, which are marked with $t_{1}, t_{2}, t_{3}$, and $t_{4}$. 
force and the fracture behavior of capped and uncapped TTR protofibrils differ. During time-force analysis, the peak force for capped protofibrils was $1400 \mathrm{pN}$, while the peak force of uncapped protofibrils was $1000 \mathrm{pN}$. Different fracture behaviors were also observed in the time-force graphs and rupture trajectories. In the case of capped protofibrils, we see only one peak force value, while uncapped protofibrils display multiple peak forces during SMD simulations. These tendencies can be supported by the rupture process as shown on the right side in Figures 3(a) and 3(b). For the capped fibrils, we see that only the top layer fractured over the 5$10 \mathrm{~ns}$ period. However, uncapped fibrils undergo multiple fracture processes and fractured beta-strands contents during this same $5-10$ ns period. Around the 3 ns period, one side of two beta-strands fractured (marked as $t_{2}$ ), with the other side fracturing around the $5.5 \mathrm{~ns}$ period (marked as $t_{3}$ ).

Similar findings resulted from the $0.005 \AA$ ps constantloading simulations as shown in Figures 3(c) and 3(d), respectively. Due to the higher constant-loading condition $(0.005$ compared to $0.001 \AA / \mathrm{ps}$ ), short fracture events were observed for both capped and uncapped protofibrils over the same time period. In addition, different fracture behavior and peak force were observed, relative to $0.001 \AA$ /ps constant-loading simulation. Only a single peak force value was observed for capped protofibrils (marked as $t_{2}$ ), while multiple peak forces were observed for uncapped TTR protofibril (denoted $t_{2}, t_{3}$, and $\left.t_{4}\right)$. The peak force difference between the two protofibril types was $800 \mathrm{pN}$, which was similar to the value from the lower loading simulation (i.e., $0.001 \AA$ /ps). Specifically, for the uncapped protofibrils, multiple fracture spots were revealed, while only the top layer was fractured in capped protofibrils. These different peak force results along with the different constant-velocity loading conditions could be compared to previous similar computational hIAPP fibril study by Choi et al. [41]. They found the different rupture force of hIAPP fibrils when different pulling speed was applied. These different rupture phenomena along with various pulling velocity also can be found in our study, where higher peak force was observed when faster pulling speed was applied [41].

Interestingly, end-terminal capping on TTR amyloid protofibrils did not affect the calculated peak force occurrence time, even under different loading and velocity conditions. Lee et al., who investigated the mechanical and material characteristics of polymorphic hIAPP protofibrils, reported that each polymorphic structure (i.e., APHO, AHPE, PHO, and PHE model) has a different peak force value with a different peak force occurrence time, based on their time-force analysis [17]. However, uncapped and capped TTR amyloid protofibrils only exhibited different peak force values, while sharing similar peak force occurrence times. Therefore, our simulations suggest that end-terminal capping only affects reaction force values, not peak force occurrence times.

Thus, from our analysis of the time-force profiles and fracture trajectories from $0.001 \AA /$ ps and $0.005 \AA /$ ps constantloading simulations, we can see that the end-terminal capping is predicted to result in different fracture behavior, albeit over similar time periods.
3.2. Fracture Characteristics of TTR Amyloid Protofibril by the End-Terminal Capping. During constant-velocity loading simulations, we found that the different fracture behavior of TTR amyloid protofibrils with different end-terminal capping status exhibited different time-force graphs and fracture trajectory profiles. In this section, we analyzed the different material fracturing behaviors of protofibrils by investigating the number of hydrogen bonds formed and the electrostatic force as a function of time. Calculating and understanding the contributions of hydrogen bonds and "nonbonding" forces such as electrostatic and van der Waals (VdW) energies are all important for assessing the stability of amyloid fibrils. For example, a previous study addressed the role of the nonbonding forces and hydrogen bonds in altering the structural conformation and mechanical properties of $\mathrm{A} \beta$ amyloid protein by mutating the leucine residue on the 19th and 20th phenylalanine residue regions [22]. Specifically, the nonbonding forces and the number of hydrogen bonds are different in each model. Notably, replacing leucine residue on the 19th phenylalanine residue region results in a lower number of hydrogen bonds, increased VdW energies, and decreased mechanical properties. Furthermore, our previous equilibrated TTR 105-115 protofibrils handled the structural stability and their electrostatic energy effect by end terminal capping [35].

In our study (see Figure 4), a constant decrease in the number of hydrogen bonds was observed under $0.001 \AA / \mathrm{ps}$ and $0.005 \AA /$ ps constant-velocity conditions. For the $0.001 \AA /$ ps constant-velocity condition, reduction in the number of hydrogen bonds stopped around the $10 \mathrm{~ns}$ period, while the loss of hydrogen bonds under $0.005 \AA /$ ps constantvelocity conditions halted around the $2 \mathrm{~ns}$ period. After the constant decrease in the number of hydrogen bonds reached a threshold, there were no additional decreases of hydrogen bonds, with the protofibrillar structures reaching a complete fracture. This tendency to decrease the number of hydrogen bonds is similar to that reported for several computational studies of amyloids using SMD simulations [17, 25, 35, 4144]. When an external force is applied to amyloid proteins, the loss of hydrogen bonds or their renewal following fracture proceeds regardless of the amyloid protein species or size. For example, our previous loading simulation study on polymorphic hIAPP fibrils showed that loss of hydrogen bonds increases in step with strain [17]. In a similar manner, Solar and Buehler reported that the incremental rupture of hydrogen bonds depends on the cross-sectional area of different amyloids (i.e., A $\beta$ and HET-s) [25]. Specifically, from our analysis of the number of hydrogen bonds over time in this study, we were unable to detect any difference in the material characteristics of capped or uncapped TTR protofibrils. According to Kim et al., hIAPP amyloid fibrils behave differently under constant bending simulations, depending on the structure of the polymorph tested [21]. Since polymorphism may alter material behavior, and TTR 105-115 does not have any polymorphs, end-terminal capping was only predicted to improve mechanical strength, not other material behaviors.

Further, we measured the variation in electrostatic force of uncapped and capped TTR protofibrils, from the initiation 


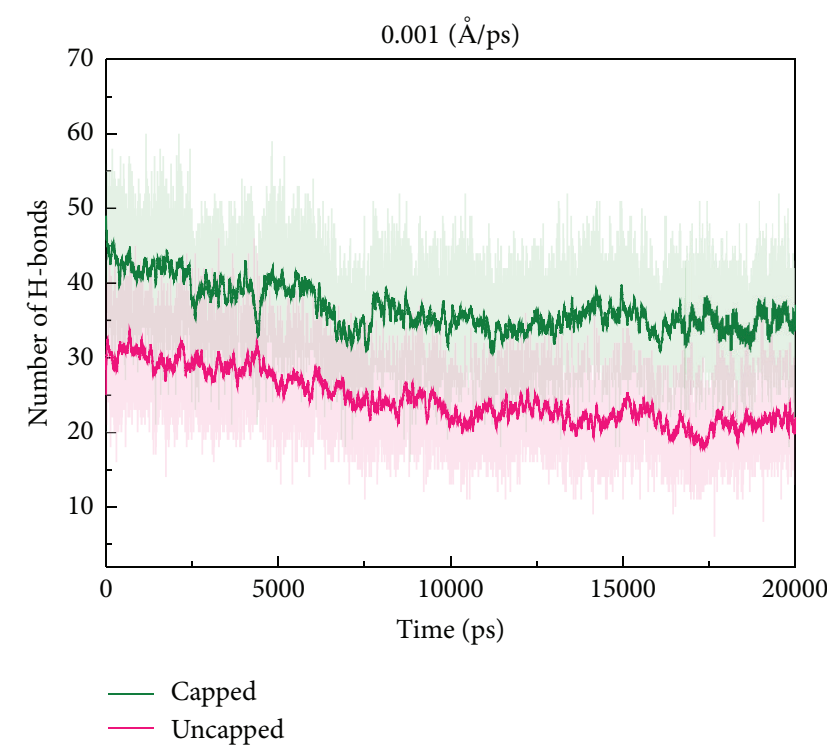

(a)

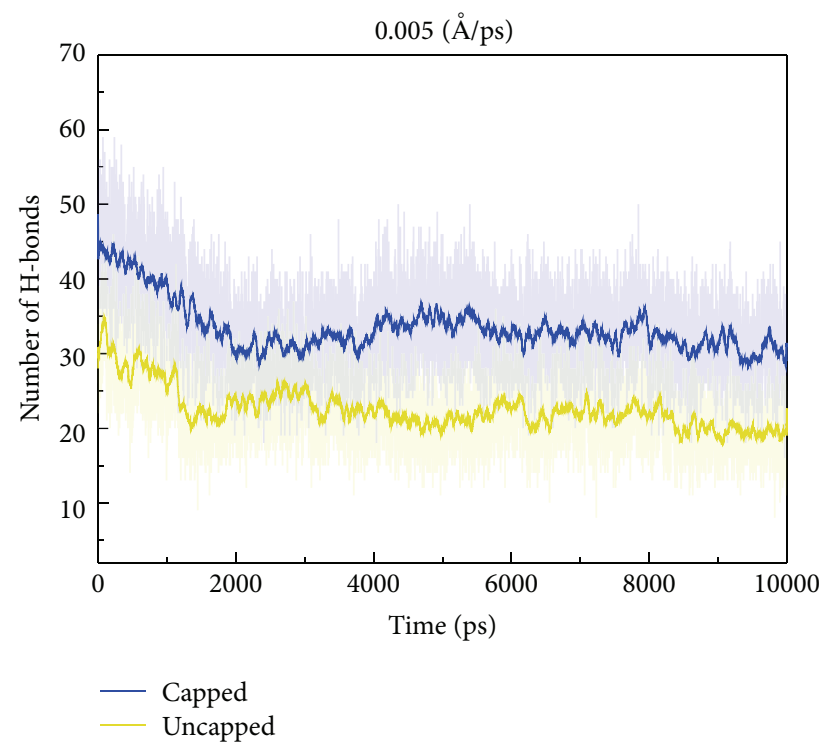

(b)

FIGURE 4: Time versus the number of hydrogen bond graphs: comparing mechanical strength differences between capped and uncapped models. (a) shows the comparison graph for the simulation performed using a velocity of $0.001 \AA / p s$, and (b) is the graph using a velocity of $0.005 \AA / \mathrm{ps}$. To reduce the noise of hydrogen bonds per time graphs, we smoothed the data using a weighted moving average with over 100 ps.

of the loading simulation to the first peak force events. In contrast, the electrostatic forces were tracked throughout the entirety of the constant-velocity loading simulations. As shown in Figures 5(a) and 5(b), electrostatic energies increased between the initiation of loading and the occurrence of the first peak force events. These tendencies are similar to those calculated for loading simulation on $\mathrm{A} \beta$ amyloid fibrils in the study by Paparcone and Buehler. They reported that the energy density of $A \beta$ amyloid fibrils increased in step with increasing strain [42]. We also calculated the stabilities of uncapped and capped TTR protofibrils via fluctuation of electrostatic forces. Our simulation shows that electrostatic energy fluctuations of uncapped fibrils were observed during both the $0.001 \AA /$ ps and the $0.005 \AA /$ ps loading conditions. In contrast, capped fibrils have fewer such fluctuations. These phenomena can be expressed as the released quantity of electrostatic energies, both uncapped and capped TTR protofibrils. Using the electrostatic energies over the whole time period, we measured the released electrostatic energy quantity of both capped and uncapped TTR protofibrils until observation of first peak force event as shown in Figure S1 in Supplementary Material available online at http:// dx.doi.org/10.1155/2016/1863065. From the result of Figure S1, we found that the electrostatic energy release of capped TTR amyloid protofibrils was constant until first peak force event for two different loading velocity conditions, which means energy release is almost zero. However, for the uncapped TTR amyloid fibrils, energy release of electrostatic energies constantly increased and their amount was occupied up to $300 \mathrm{kcal} / \mathrm{mol}$. Specifically, we calculated the fluctuation of electrostatic energies by measuring the standard deviation as shown in Figure 5(c), where the standard deviation of electrostatic forces is represented as error bars of average electrostatic forces. From the figure, a large-value error bar of uncapped TTR protofibril was observed, while a small-value error bar of capped TTR protofibril was observed. Therefore, end-terminal capping on TTR protofibrils not only lowers the electrostatic forces but also strengthens and lowers the electrostatic energies fluctuations.

Our analysis of hydrogen bond prevalence, together with our analysis of electrostatic energies, supports our earlier results that end-terminal capping of TTR protofibrils effects an increase in disruptive forces, resulting in a lower fraction the beta-strands contents compared to uncapped protofibrils.

3.3. Material Properties of Capped and Uncapped TTR Amyloid Protofibrils. Based on time-force analysis, we assessed the mechanical properties of capped and uncapped TTR amyloid protofibrils by generating stress-strain curves. Stressstrain curves for our in silico protofibrils were produced by calculating the strain and using Hooke's law as described in Section 2.3. The stress-strain graphs are depicted in Figure 6. As can be seen in Figure 6(a), under $0.001 \AA /$ ps constantvelocity loading simulation, capped protofibrils have higher stress values than uncapped protofibrils at around 0.5 strain regions. The calculated strain differs under the velocity loading condition of $0.005 \AA / \mathrm{ps}$, as shown in Figure 6(b). Using this stress-strain relationship, we calculated Young's modulus, as shown in Figure 6(c). Under both loading simulation conditions $(0.001 \AA / \mathrm{ps}$ and $0.005 \AA / \mathrm{ps})$, we can saw that Young's modulus of capped protofibrils $(7-8 \mathrm{GPa})$ was larger than that of uncapped protofibrils $(6-6.5 \mathrm{GPa})$. These results are consistent with our previous equilibrated MD and PCA analysis results, in which the axial elastic modulus of capped TTR protofibrils was higher than the uncapped TTR protofibrils. Furthermore, Young's modulus 


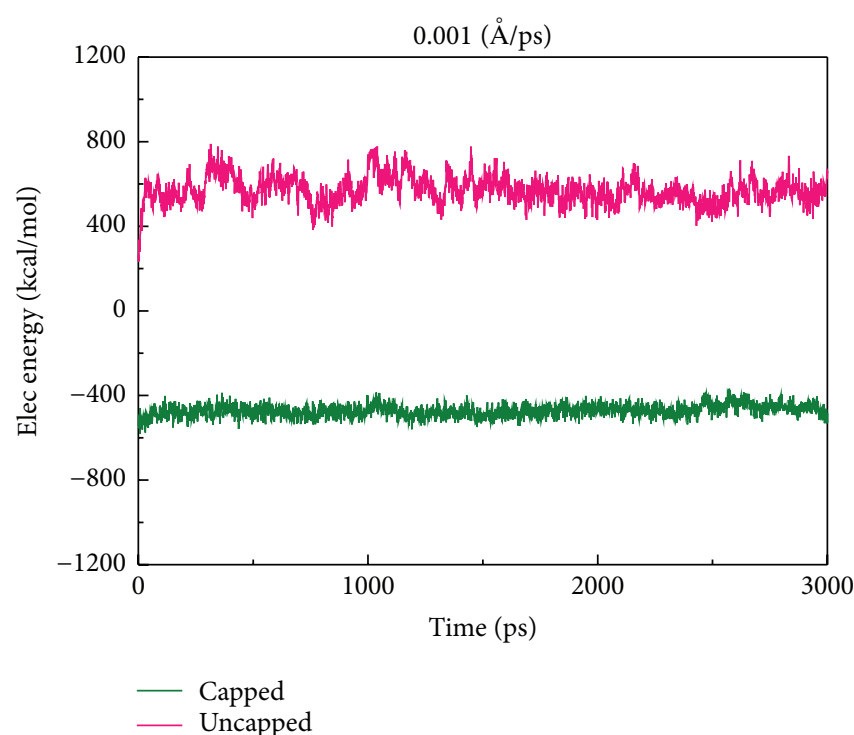

(a)

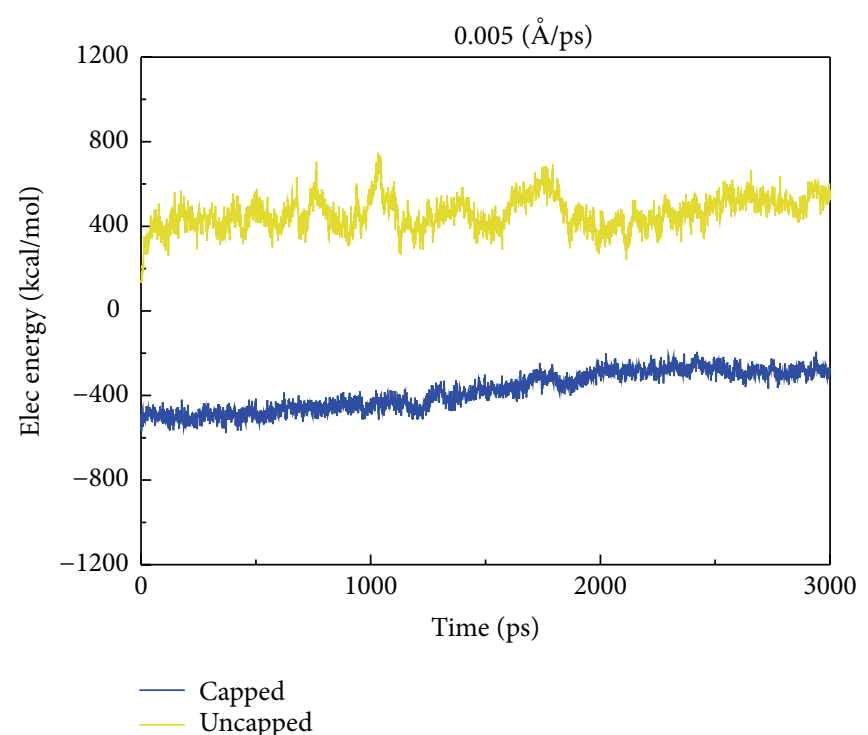

(b)

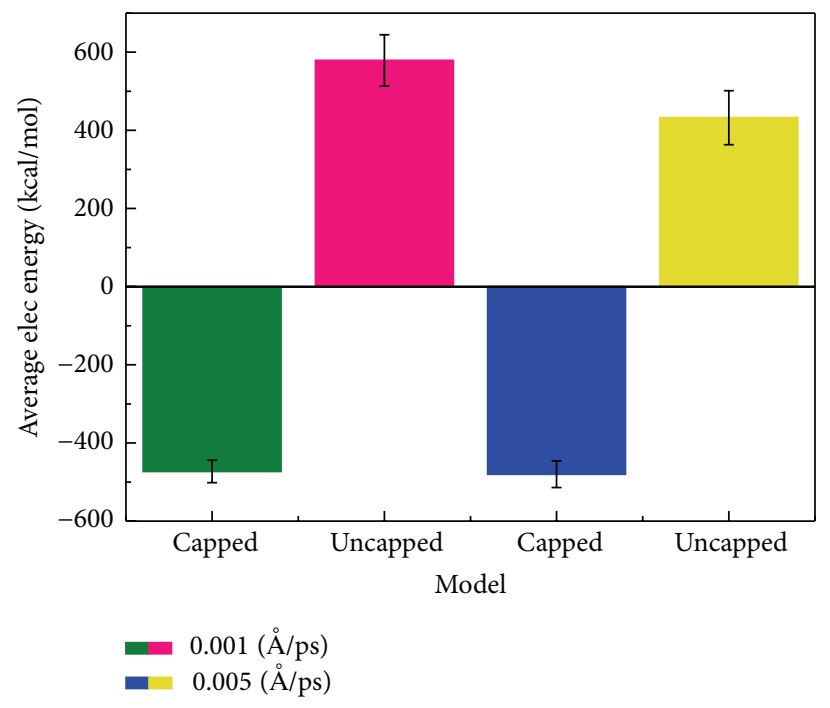

(c)

FIGURE 5: Time versus electrostatic energy graphs: comparing the structural characteristics effect of end-terminal capping on TTR (105-115) protofibrils. (a) shows the comparison graph for the simulation performed using a velocity of $0.001 \AA / p s$, (b) shows the graph using a velocity of $0.005 \AA / p s$, and (c) shows the average number of electrostatic energies. Error bars represent the standard deviations.

difference of capped and uncapped TTR amyloid protofibrils with respect to various pulling speed also can be compared to bending simulation on hIAPP fibrils along with various length size [41]. From their result of bending and shear stress, both stresses were increased when pulling speed also increased. Considering the similar elastic modulus results obtained in this study, our Young's modulus of TTR amyloid protofibrils is higher when applied $0.005 \AA / p s$ than those of $0.001 \AA / p s$. That means that higher mechanical response of amyloid fibril comes from the faster pulling speed.

Young's modulus values we obtained using SMD simulations can be compared to previous experimental studies on TTR 105-115 fibrils. Meersman et al. reported that the bulk modulus of TTR 105-115 fibril is $2.6 \mathrm{GPa}$ [45]. Considering that our capped and uncapped fibrils are nanoscale structures, our calculated material properties are reliable results in terms of having a similar degree of order. Our Young's modulus results can also be compared to the results obtained for different amyloid proteins, using both experimental and computational methods. As shown in Table 1, our results have a similar Young's modulus degree of order compared to previously studied amyloid, despite differences in the underlying amyloid proteins. When compared to the "parallel/homo" polymorph structure of hIAPP fibrils, our structure is predicted to have a higher Young's modulus $[17,18,21]$. In addition, our simulations suggest that both 


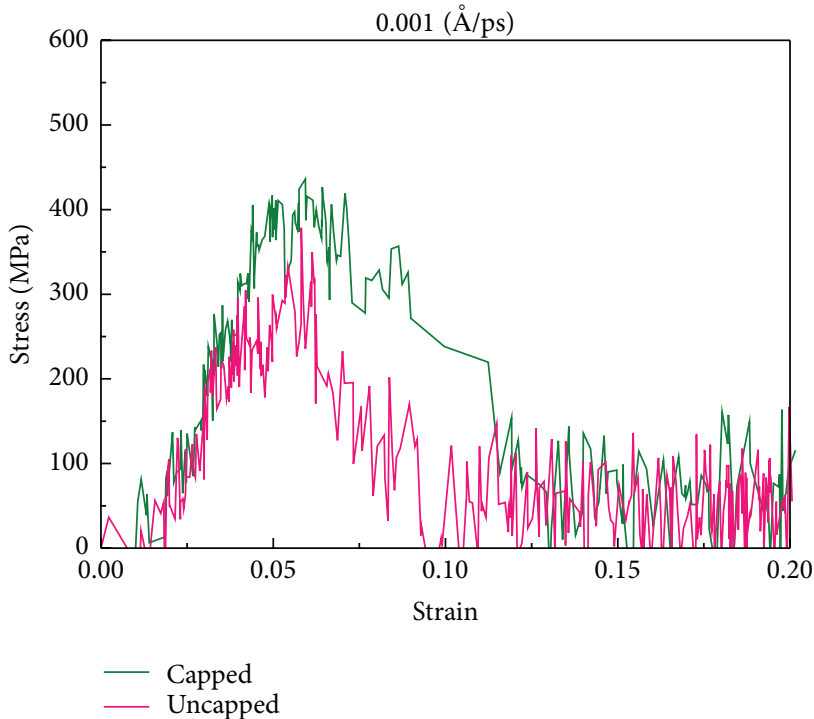

(a)

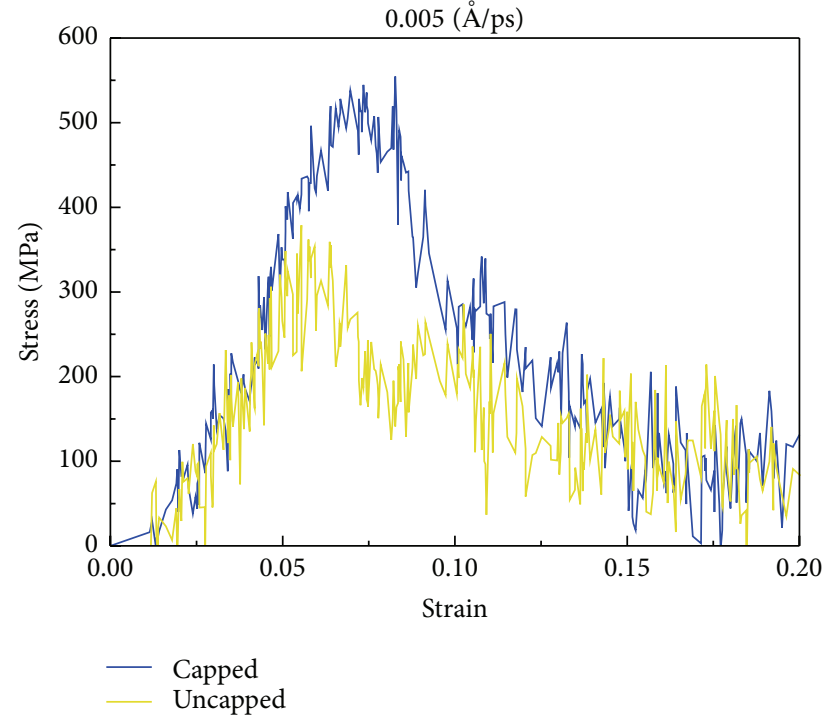

(b)

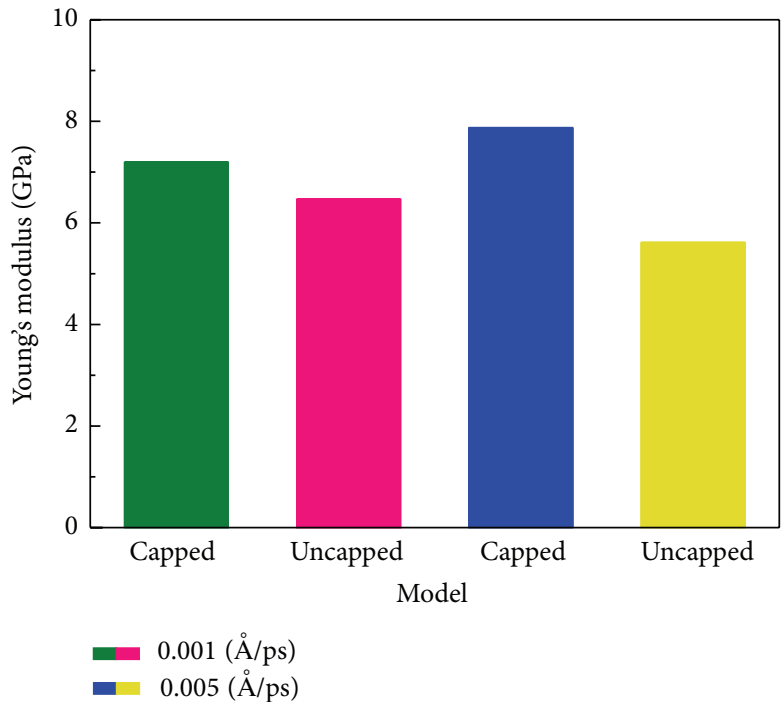

(c)

FIgURE 6: Stress versus strain graphs and Young's modulus graphs. Stress-strain curves for each pulling rate $(0.001 \AA / \mathrm{ps}$ and $0.005 \AA / \mathrm{ps})$ to compute Young's modulus for both capped and uncapped fibrils. (a) Strain-stress curve calculated from pulling rate of $0.001 \AA / \mathrm{ps}$, (b) strain-stress curve obtained from pulling rate of $0.005 \AA / \mathrm{ps}$, and (c) Young's modulus of uncapped and capped TTR 105-115 protofibrils from stress-strain curves.

TABLE 1: The tensile Young's modulus of several amyloid fibrils for the comparison.

\begin{tabular}{lc}
\hline Structures & Elastic modulus (GPa) \\
\hline hIAPP (SMD) [17, 18] & $2.4-12$ \\
hIAPP (ENM) [19] & $12-14$ \\
A $\beta$ (SMD) [42-44] & $12.4-17.2$ \\
TTR (experiment) [45] & 2.6 \\
TTR (our study) & $6-8$ \\
\hline
\end{tabular}

uncapped and capped TTR 105-115 protofibrils have a higher Young's modulus than insulin amyloid fibrils.

From our computational stress-strain and Young's modulus analyses, we predict that end-terminal capping of TTR
105-115 protofibril results in material with a higher Young's modulus than uncapped protofibrils. Our results including time-force graphs, fracture trajectory analysis, the number of hydrogen bonds, and electrostatic force analysis due to the end-terminal capping effects are consistent with previous similar amyloid studies. Further, our results show the reliable data compared to previous experimental and computational TTR 105-115 amyloid fibril studies.

\section{Conclusions}

In this study, we calculated material properties of capped and uncapped TTR (105-115) protofibrils by investigating 
their structural and mechanical response to different loadings at constant-velocity, using steered molecular dynamics. Analysis of the time-force and rupture trajectory profiles showed that capped protofibrils have a greater rupture force but lower ruptured beta-strand contents than uncapped protofibrils. These profiles were consistent with an analysis of electrostatic energy fluctuations and the number of the hydrogen bonds. During the fracture process, we found larger electrostatic force fluctuations in uncapped protofibrils than capped protofibrils. Further, we computationally derived and predicted the material properties of the protofibrils from our SMD simulations. Stress-strain curves indicated that capped protofibrils are likely to have a larger Young's modulus $(7-8 \mathrm{GPa})$ than uncapped protofibrils $(6-6.5 \mathrm{GPa})$. Understanding the mechanical characteristics of amyloid protofibrils is important, since their growth mechanism relies on fragmentation and elongation. Our study provides a useful model of the effect of end-terminal capping on TTR 105115 amyloid protofibrils. In particular, since amyloid fibrils are increasingly being used as basic templates for functional material [46], our study suggests an additional material design methodology for templating biofunctional materials.

\section{Competing Interests}

The authors declare that there is no conflict of interests regarding the publication of this paper.

\section{Authors' Contributions}

Myeongsang Lee and Hyunsung Choi made equal contribution.

\section{Acknowledgments}

Sungsoo Na gratefully acknowledges the Basic Science Research Program, through the National Research Foundation of Korea (NRF), and is funded by the Ministry of Science, ICT \& Future Planning (MSIP) (Grant no. 2014R1A2A1A11052389).

\section{References}

[1] G. Merlini and V. Bellotti, "Molecular mechanisms of amyloidosis," The New England Journal of Medicine, vol. 349, no. 6, pp. 583-596, 2003.

[2] C. Soto, "Unfolding the role of protein misfolding in neurodegenerative diseases," Nature Reviews Neuroscience, vol. 4, no. 1, pp. 49-60, 2003.

[3] F. Chiti and C. M. Dobson, "Protein misfolding, functional amyloid, and human disease," Annual Review of Biochemistry, vol. 75, pp. 333-366, 2006.

[4] J. W. M. Höppener, B. Ahrén, and C. J. M. Lips, "Islet amyloid and type 2 diabetes mellitus," The New England Journal of Medicine, vol. 343, no. 6, pp. 411-419, 2000.

[5] N. M. Kad, S. L. Myers, D. P. Smith, D. A. Smith, S. E. Radford, and N. H. Thomson, "Hierarchical assembly of $\beta 2$-microglobulin amyloid in vitro revealed by atomic force microscopy," Journal of molecular biology, vol. 330, no. 4, pp. 785-797, 2003.

[6] N. M. Kad, N. H. Thomson, D. P. Smith, D. A. Smith, and S. E. Radford, " $\beta 2$-microglobulin and its deamidated variant, N17D form amyloid fibrils with a range of morphologies in vitro," Journal of Molecular Biology, vol. 313, no. 3, pp. 559-571, 2001.

[7] C. Rapezzi, C. C. Quarta, L. Riva et al., "Transthyretin-related amyloidoses and the heart: a clinical overview," Nature Reviews Cardiology, vol. 7, no. 7, pp. 398-408, 2010.

[8] J. N. Dungu, L. J. Anderson, C. J. Whelan, and P. N. Hawkins, "Cardiac transthyretin amyloidosis," Heart, vol. 98, no. 21, pp. 1546-1554, 2012.

[9] T. P. J. Knowles and M. J. Buehler, "Nanomechanics of functional and pathological amyloid materials," Nature Nanotechnology, vol. 6, no. 8, pp. 469-479, 2011.

[10] M. J. Buehler and Y. C. Yung, "Deformation and failure of protein materials in physiologically extreme conditions and disease," Nature Materials, vol. 8, no. 3, pp. 175-188, 2009.

[11] C. Haass and D. J. Selkoe, "Soluble protein oligomers in neurodegeneration: lessons from the Alzheimer's amyloid $\beta$ peptide," Nature Reviews Molecular Cell Biology, vol. 8, no. 2, pp. 101-112, 2007.

[12] S. A. Kotler, P. Walsh, J. R. Brender, and A. Ramamoorthy, "Differences between amyloid- $\beta$ aggregation in solution and on the membrane: insights into elucidation of the mechanistic details of Alzheimer's disease," Chemical Society Reviews, vol. 43, no. 19, pp. 6692-6700, 2014.

[13] J. R. Brender, D. L. Heyl, S. Samisetti et al., "Membrane disordering is not sufficient for membrane permeabilization by islet amyloid polypeptide: studies of IAPP(20-29) fragments," Physical Chemistry Chemical Physics, vol. 15, no. 23, pp. 89088915, 2013.

[14] J. R. Brender, S. Salamekh, and A. Ramamoorthy, "Membrane disruption and early events in the aggregation of the diabetes related peptide IAPP from a molecular perspective," Accounts of Chemical Research, vol. 45, no. 3, pp. 454-462, 2012.

[15] S. R. Collins, A. Douglass, R. D. Vale, and J. S. Weissman, "Mechanism of prion propagation: amyloid growth occurs by monomer addition," PLoS Biology, vol. 2, article e321, 2004.

[16] J. F. Smith, T. P. J. Knowles, C. M. Dobson, C. E. MacPhee, and M. E. Welland, "Characterization of the nanoscale properties of individual amyloid fibrils," Proceedings of the National Academy of Sciences of the United States of America, vol. 103, no. 43, pp. 15806-15811, 2006.

[17] M. Lee, H. J. Chang, D. Kim et al., "Relationship between structural composition and material properties of polymorphic hIAPP fibrils," Biophysical Chemistry, vol. 199, pp. 1-8, 2015.

[18] M. Lee, I. Baek, H. J. Chang, G. Yoon, and S. Na, "The bond survival time variation of polymorphic amyloid fibrils in the mechanical insight," Chemical Physics Letters, vol. 600, pp. 6872, 2014.

[19] G. Yoon, J. Kwak, J. I. Kim, S. Na, and K. Eom, "Mechanical characterization of amyloid fibrils using coarse-grained normal mode analysis," Advanced Functional Materials, vol. 21, no. 18, pp. 3454-3463, 2011.

[20] G. Yoon, Y. K. Kim, K. Eom, and S. Na, "Relationship between disease-specific structures of amyloid fibrils and their mechanical properties," Applied Physics Letters, vol. 102, Article ID 011914, 2013.

[21] J. I. Kim, M. Lee, I. Baek, G. Yoon, and S. Na, "The mechanical response of hIAPP nanowires based on different bending 
direction simulations," Physical Chemistry Chemical Physics, vol. 16, no. 34, pp. 18493-18500, 2014.

[22] H. J. Chang, I. Baek, M. Lee, and S. Na, "Influence of aromatic residues on the material characteristics of $\mathrm{A} \beta$ amyloid protofibrils at the atomic scale," ChemPhysChem, vol. 16, no. 11, pp. 2403-2414, 2015.

[23] R. Paparcone, M. A. Pires, and M. J. Buehler, "Mutations alter the geometry and mechanical properties of Alzheimer's $\mathrm{A} \beta(1-$ 40) amyloid fibrils," Biochemistry, vol. 49, no. 41, pp. 8967-8977, 2010.

[24] M. Lee, I. Baek, H. Choi, J. I. Kim, and S. Na, "Effects of lysine residues on structural characteristics and stability of tau proteins," Biochemical and Biophysical Research Communications, vol. 466, no. 3, pp. 486-492, 2015.

[25] M. Solar and M. J. Buehler, "Tensile deformation and failure of amyloid and amyloid-like protein fibrils," Nanotechnology, vol. 25, no. 10, Article ID 105703, 2014.

[26] Z. Xu, R. Paparcone, and M. J. Buehler, “Alzheimer's A $\beta(1-40)$ amyloid fibrils feature size-dependent mechanical properties," Biophysical Journal, vol. 98, no. 10, pp. 2053-2062, 2010.

[27] H. Choi, H. J. Chang, Y. Shin et al., "The molecular mechanism of conformational changes of the triplet prion fibrils for $\mathrm{pH}$," RSC Advances, vol. 5, no. 61, pp. 49263-49269, 2015.

[28] H. Ndlovu, A. E. Ashcroft, S. E. Radford, and S. A. Harris, "Molecular dynamics simulations of mechanical failure in polymorphic arrangements of amyloid fibrils containing structural defects," Beilstein Journal of Nanotechnology, vol. 4, no. 1, pp. 429-440, 2013.

[29] H. Ndlovu, A. E. Ashcroft, S. E. Radford, and S. A. Harris, "Effect of sequence variation on the mechanical response of amyloid fibrils probed by steered molecular dynamics simulation," Biophysical Journal, vol. 102, no. 3, pp. 587-596, 2012.

[30] G. Yoon, M. Lee, J. I. Kim, S. Na, and K. Eom, "Role of sequence and structural polymorphism on the mechanical properties of amyloid fibrils," PLoS ONE, vol. 9, no. 2, Article ID e88502, 2014.

[31] M. Porrini, U. Zachariae, P. E. Barran, and C. E. MacPhee, "Effect of protonation state on the stability of amyloid oligomers assembled from TTR(105-115)," The Journal of Physical Chemistry Letters, vol. 4, no. 8, pp. 1233-1238, 2013.

[32] M. Andreasen, K. K. Skeby, S. Zhang et al., "The importance of being capped: terminal capping of an amyloidogenic peptide affects fibrillation propensity and fibril morphology," Biochemistry, vol. 53, no. 44, pp. 6968-6980, 2014.

[33] K. Tao, J. Wang, P. Zhou et al., "Self-assembly of short A $\beta(16-22)$ peptides: effect of terminal capping and the role of electrostatic interaction," Langmuir, vol. 27, no. 6, pp. 2723-2730, 2011.

[34] V. Castelletto, I. W. Hamley, Ç. Cenker et al., "Influence of end-capping on the self-assembly of model amyloid peptide fragments," Journal of Physical Chemistry B, vol. 115, no. 9, pp. 2107-2116, 2011.

[35] M. Lee and S. Na, "End capping alters the structural characteristics and mechanical properties of transthyretin (105-115) amyloid protofibrils," ChemPhysChem, vol. 17, no. 3, pp. 425432, 2016.

[36] A. W. P. Fitzpatrick, G. T. Debelouchina, M. J. Bayro et al., "Atomic structure and hierarchical assembly of a cross- $\beta$ amyloid fibril," Proceedings of the National Academy of Sciences of the United States of America, vol. 110, no. 14, pp. 5468-5473, 2013.

[37] C. P. Jaroniec, C. E. MacPhee, V. S. Bajaj, M. T. McMahon, C. M. Dobson, and R. G. Griffin, "High-resolution molecular structure of a peptide in an amyloid fibril determined by magic angle spinning NMR spectroscopy," Proceedings of the National Academy of Sciences of the United States of America, vol. 101, no. 3, pp. 711-716, 2004.

[38] C. P. Jaroniec, C. E. MacPhee, N. S. Astrof, C. M. Dobson, and R. G. Griffin, "Molecular conformation of a peptide fragment of transthyretin in an amyloid fibril," Proceedings of the National Academy of Sciences of the United States of America, vol. 99, no. 26, pp. 16748-16753, 2002.

[39] W. Humphrey, A. Dalke, and K. Schulten, "VMD: visual molecular dynamics," Journal of Molecular Graphics, vol. 14, no. 1, pp. 33-38, 1996.

[40] J. C. Phillips, R. Braun, W. Wang et al., "Scalable molecular dynamics with NAMD," Journal of Computational Chemistry, vol. 26, no. 16, pp. 1781-1802, 2005.

[41] B. Choi, G. Yoon, S. W. Lee, and K. Eom, "Mechanical deformation mechanisms and properties of amyloid fibrils," Physical Chemistry Chemical Physics, vol. 17, no. 2, pp. 1379-1389, 2015.

[42] R. Paparcone and M. J. Buehler, "Failure of A $\beta(1-40)$ amyloid fibrils under tensile loading," Biomaterials, vol. 32, no. 13, pp. 3367-3374, 2011.

[43] R. Paparcone, S. Keten, and M. J. Buehler, "Atomistic simulation of nanomechanical properties of Alzheimer's AB(1-40) amyloid fibrils under compressive and tensile loading," Journal of Biomechanics, vol. 43, no. 6, pp. 1196-1201, 2010.

[44] R. Paparcone, S. Keten, and M. Buehler, "Amyloid nanofibrils under compressive loading," Journal of Biomechanics, vol. 43, pp. 1196-1201, 2009.

[45] F. Meersman, R. Q. Cabrera, P. F. McMillan, and V. Dmitriev, "Structural and mechanical properties of TTR105-115 amyloid fibrils from compression experiments," Biophysical Journal, vol. 100, no. 1, pp. 193-197, 2011.

[46] I. Cherny and E. Gazit, "Amyloids: not only pathological agents but also ordered nanomaterials," Angewandte ChemieInternational Edition, vol. 47, no. 22, pp. 4062-4069, 2008. 

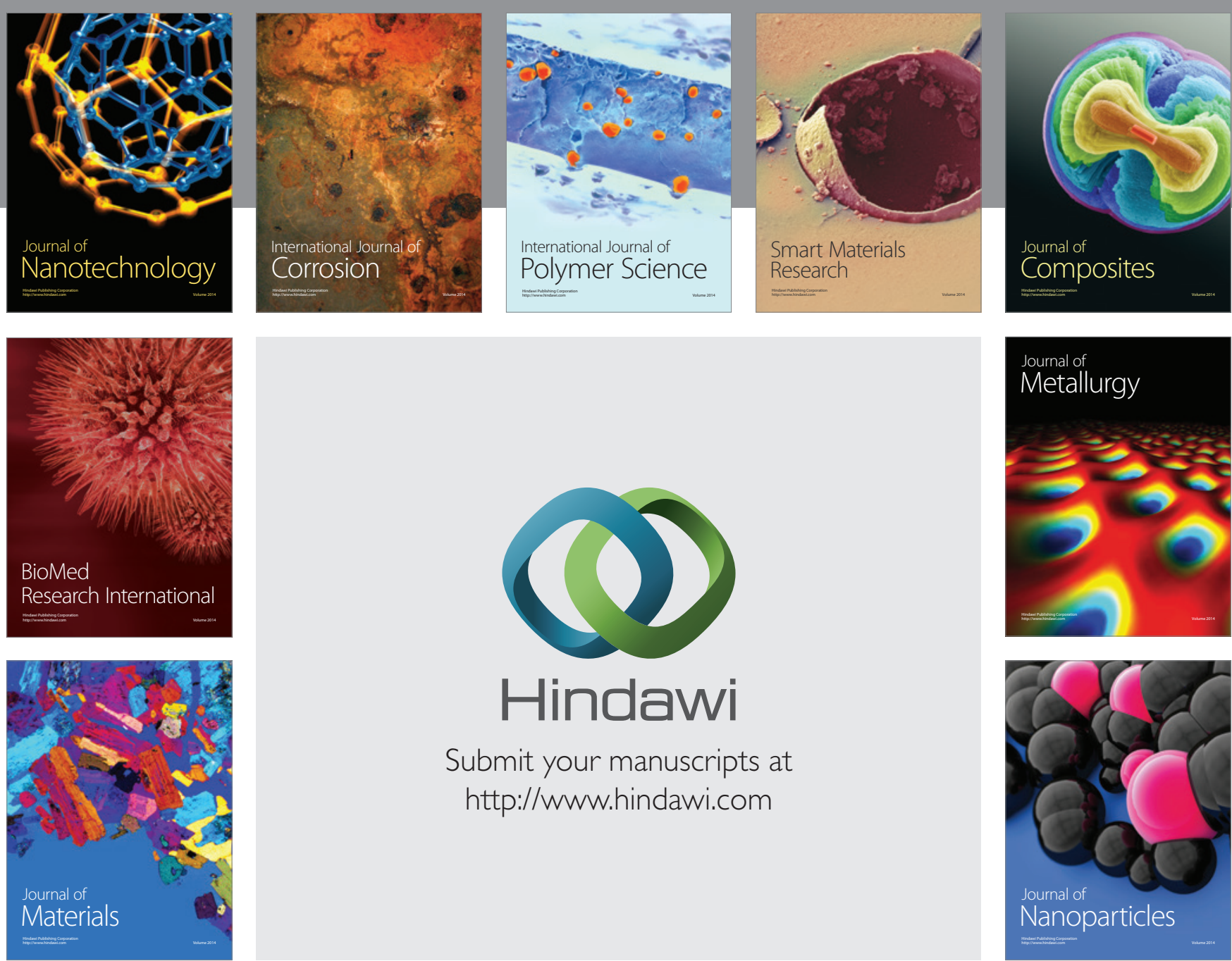

\section{Hindawi}

Submit your manuscripts at

http://www.hindawi.com

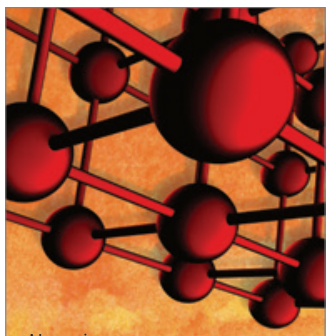

Materials Science and Engineering
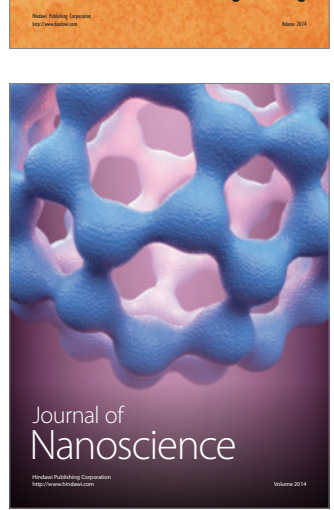
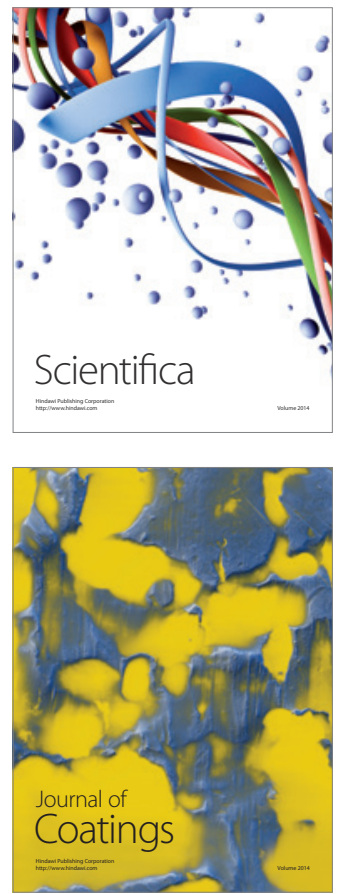
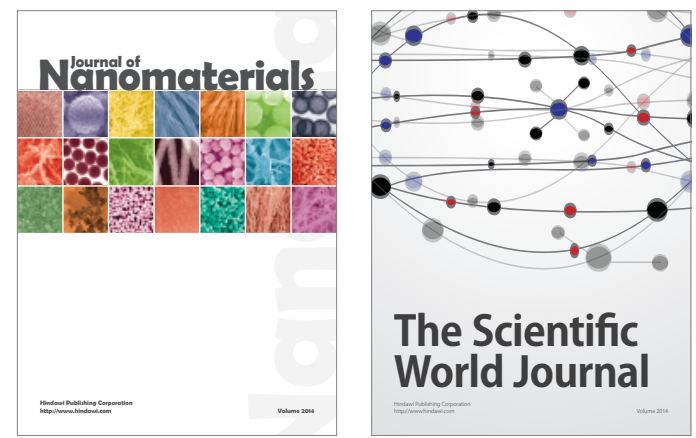

The Scientific World Journal
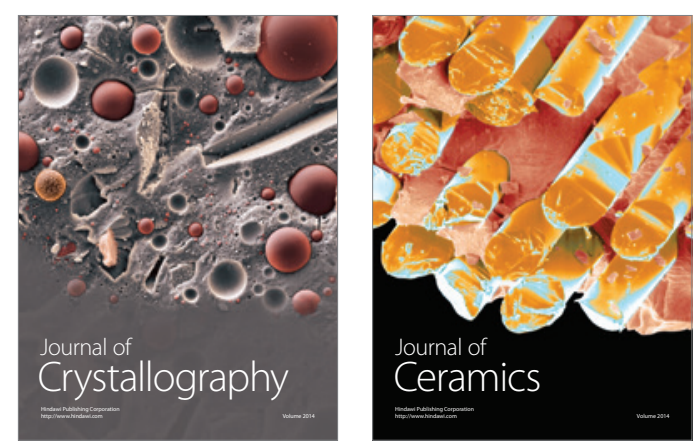
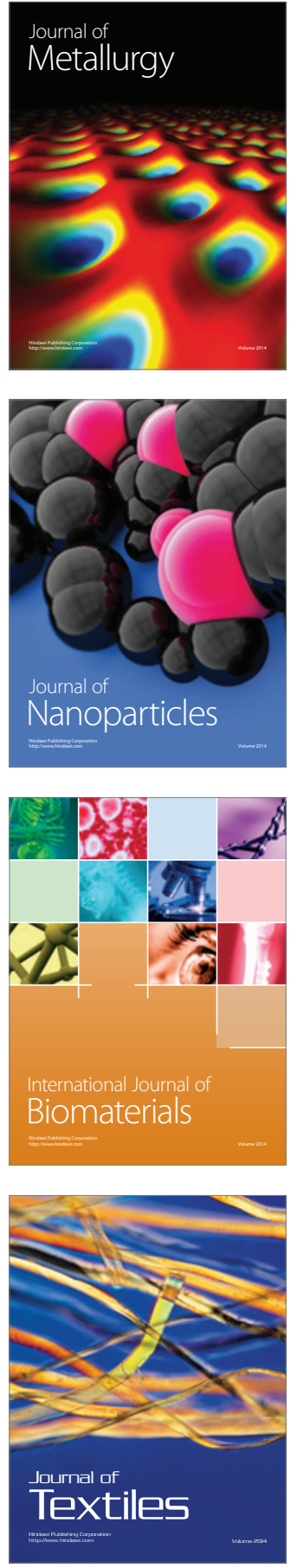